\title{
Editorial
}

\section{Preface to Special Column on Electrocatalysis}

Energy is the most important problem that we are facing. The limited fossil fuel reserves and the air pollution caused by the consuming of fossil fuels force the governments, industries and academia to look for renewable energies and their conversion-storage devices. Some novel concepts based on the electrochemical reactions have been considered as promising solutions to help solve energy problems and improve the quality of our lives. Electrocatalysis plays a critical role in the advanced electrochemical energy systems such as fuell cells, metal-air batteries, and electrolyzers..

This Special Column aims to cover recent progress and trends in advanced electrocatalysts for various electrochemical reactions including oxygen reduction reaction, hydrogen oxidation and evolution reactions, oxygen evolution reaction, and methanol oxidation. It includes 12 high quality papers, among which 6 are reviews and perspective. Contributors are from China, North America, Europe, and South Korea. We would like to thank all the authors for their great contributions and referees for their time reviewing the manuscripts. I believe these excellent papers collected in this Special Column will make significant contributions to the electrocatalysis community.

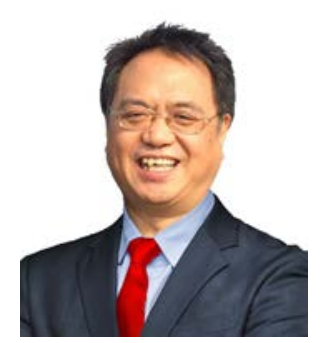

Prof. Zidong Wei

School of Chemistry and Chemical Engineering, Chongqing University, Shapingba 174, Chongqing 400044, China

E-mail: zdwei@cqu.edu.cn

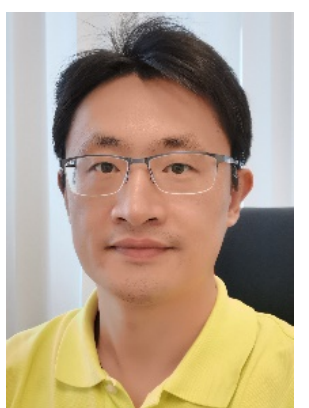

Prof. Minhua Shao

Department of Chemical and Biological Engineering, Hong Kong University of Science and Technology, China

E-mail: kemshao@ust.hk

DOI: 10.1016/S1872-2067(20)63558-6| http://www.sciencedirect.com/science/journal/18722067 | Chin. J. Catal., Vol. 41, No. 5, May 2020 (C) 2020, Dalian Institute of Chemical Physics, Chinese Academy of Sciences. Published by Elsevier B.V. All rights reserved. 